\title{
Role of Diffusion-weighted Magnetic Resonance Imaging in Cervical Cancer for Prediction and Monitoring of Chemo-radiotherapy Response
}

\author{
Prachi Kala*, Vidya Bhargavi, Rohini Avantsa, Geeta Narayan \\ Department of Radiology and Imaging, Vydehi Institute of Medical Sciences and Research Centre, India
}

Copyright $\mathrm{C} 2017$ by authors, all rights reserved. Authors agree that this article remains permanently open access under the terms of the Creative Commons Attribution License 4.0 International License

\begin{abstract}
Objectives: To investigate the possibility of DWI as an imaging bio marker and evaluate its role in prediction and monitoring of chemoradiotherapy response in cervical carcinoma. Methods: 30 carcinoma cervix patients undergoing radiation for Carcinoma Cervix were examined with routine pelvic MRI and DWI before chemoradiation, following EBRT and on completion of brachytherapy. Based on the response on conventional MRI patients were categorized into complete, good and partial response groups. Analysis of variance (ANOVA) and Student $t$ test (two tailed, independent) were used to compare the ADC, size and volume parameters between response groups and therapeutic times. Pearson's correlation test was calculated between ADC parameters and tumor size and volume response. Results: Cervical cancer demonstrated serial increase in tumor ADC values with corresponding decrease in tumor diameters and volumes during the therapy (all $\mathrm{p}$ values $<0.001$ ). significant and moderate positive linear correlation was found between the tumor ADC values and final size and volume responses at pre-treatment, post EBRT and post-treatment. Tumors with high diffusion values responded better to therapy than with low diffusion values. Patients with greater post EBRT tumor ADC changes and greater size and volume responses responded better to therapy. The pretreatment tumor ADC values, post EBRT ADC changes were found to be correlating well with the final outcome. However ADC mapping was less useful in predicting the extension and staging of carcinoma. Conclusion: DWI and its quantitative parameter, $\mathrm{ADC}$ has a potential role in the prediction, assessment and monitoring of cervical cancer treatment response to chemo radiation therapy.
\end{abstract}

Keywords MRI: Magnetic Resonance Imaging, DWI: Diffusion Weighted Imaging, EBRT: External Beam Radio-therapy, Carcinoma Cervix, Chemoradioherapy

\section{Introduction}

Of all the imaging modalities, Magnetic resonance imaging (MRI) is most widely used in the evaluation of cervical cancer. Precise assessment of tumor stage in uterine cervical cancer is necessary to determine the treatment of choice. MRI is considered the best diagnostic method for assessing the stage of cervical cancer because of its better spatial and contrast resolution with the possibility of multidimensional imaging [1]. However its role in evaluation of cervical cancer is insufficient due to the limitations of morphologic imaging, particularly in lesion characterization, accurate lymph node staging, assessment of tumor response and inability to differentiate post treatment changes from tumor recurrence [2]

Diffusion weighted MRI has added a new dimension in treatment planning and post treatment changes $[3,4]$.

Due to tumour heterogeneity, not all cancers respond to a specific therapy and once the treatment fails further therapeutic options become limited and result in unnecessary patient morbidity and mortality. Development of an MR imaging biomarker that would allow for early and reliable prediction of tumour response to therapeutic intervention would be a significant achievement as it could individualize clinical management of cancer patients in a timely fashion and improve outcome.

Diffusion weighted magnetic resonance imaging (DW-MRI) is a non-invasive functional imaging technique that can be used to quantify the thermal motion of water molecules in tumour tissue. This is sensitive to the underlying tumour microenvironment which can make subtle abnormality more obvious and can provide better characterization of tissue and their pathological processes at microscopic level. Since the morphologic changes in gross tumour size lag behind the biological and molecular changes following therapy, diffusion has the potential to provide feedback related to the treatment effects over time. In addition, it allows quantification of the treatment induced 
changes which may occur before conventional morphologic alterations [5]. Thus, the response to therapy can be assessed both qualitatively by inspecting high signal intensity on high b-value DW images and quantitatively with apparent diffusion co-efficient (ADC) measurements. Changes in tumour ADC values during treatment have been shown to correlate with outcome, suggesting a role for DW-MRI as a predictive imaging biomarker of early response. The measurements can be obtained without the need for exogenous contrast medium administration. Finally, DW-MRI can be acquired in a relatively short period of time.

The motivation for developing a validated imaging biomarker of treatment response is in part due to its potential to noninvasively and repeatedly interrogate the entire tumour mass over time (or in the case of metastatic disease multiple tumours at once)[6]

Overall, these features provide DW-MRI with the potential to provide for a standardized imaging measurement in the evaluation of therapeutic response. But also there is a need to further carefully investigate the possible advantages of DWI as an imaging biomarker and evaluate for its ability to accurately measure tumour response in cervical carcinoma of uterus.

\section{Methods and Materials}

\subsection{Study Population}

Thirty female patients ranging in age from 30 to 70 years with suspicious uterine cervical lesions and known uterine cervical carcinoma are clinically staged by oncologist from department of oncology in our institution and referred to our Department of radiology, Vydehi institute of medical sciences, Bangalore for MR examination (pre-treatment, intra-treatment and post treatment) between October 2012 and May 2013.

Patients with histopathologically proved and clinically staged cervical carcinoma were included. Patients with contra indications to MRI were excluded.

All patients were treated with concurrent chemoradiotherapy. Concurrent chemo radiotherapy comprised of pelvic external beam radiotherapy of $4600 \mathrm{cGy}$ in 23 fractions, 200 cGy per fraction, 5 fractions per week to whole pelvis and three applications of high dose rate intracavitary brachytherapy (700cGy/fraction) combined with Inj Cisplatin $\left(40 \mathrm{mg} / \mathrm{m}^{2}\right)$ weekly, total of 5 cycles.

All patients underwent routine pelvic Magnetic resonance imaging and transverse diffusion weighted imaging before initiation of chemo radiation, during therapy following completion of external beam radiotherapy (EBRT) and after therapy following completion of brachytherapy with a $1.5-\mathrm{T}$ MR scanner and a 16 channel torso coil.

\subsection{MR Imaging}

MR examinations were performed on a 1.5 Tesla Philips Achieva scanner (Philips Medical system, Netherlands) using a 16 channel SENSE XL torso coil. T1, T2, and post contrast $\mathrm{T} 1$ pulse sequences were acquired for the cervix with coronal sections parallel to the long axis of cervix and axial sections perpendicular to the long axis of cervix. Axial Diffusion sequences were obtained both for $b-0$ and b-1000 (TR/TE $=1296 / 59 \mathrm{~mm}$ ) with $305 \mathrm{~mm}$ FOV, 124 X 100 matrix, $5 \mathrm{~mm}$ slice thickness and $1 \mathrm{~mm}$ interslice gap.

\subsection{Image Analysis}

The tumor size and volume were measured at each therapeutic time based on conventional T2W images. Images were also studied for tumor extension and staging.

Final or post EBRT tumor size or volume reduction (\%) was calculated using the equation:

Tumor reduction $\%=\frac{\{\text { Pretreatment diameter /volume }- \text { post EBRT or post treatment diameter /volume }\}}{\text { Pretreatment diameter /volume }} \times 100$

The ADC values from diffusion sequences were measured and compared at all three stages of study: Pretreatment, post EBRT and post treatment. The apparent diffusion coefficient (ADC) values in the tumor, normal gluteus muscle and normal rectal mucosa were automatically calculated by manual placement of the region of interest (ROI).

Post EBRT or Final change in tumor ADC values (\%) were calculated using the equation:

$$
\% \text { ADC change }=\frac{\text { Post treatment/post EBRT ADC }- \text { pretreatment ADC }}{\text { Pretreatment ADC }} \times 100
$$

The response to treatment was determined by comparing conventional MR images at all treatment stages. Based on the reduction of tumor size patients were categorized into complete, good and partial response groups. Patients with no residual tumor were considered as complete response group. Patients with more than $60 \%$ of tumor size reduction were concluded as good response group and those with less than $60 \%$ as partial response group. 
Table 1. Pre-treatment, post EBRT and post treatment comparative response evaluation of tumour size, volume and ADC

\begin{tabular}{|c|c|c|c|c|c|c|c|}
\hline & Pre-Tx & Post EBRT & Post-Tx & $\begin{array}{c}\text { Post EBRT \% } \\
\text { change }\end{array}$ & $\begin{array}{c}\text { Post Tx \% } \\
\text { change }\end{array}$ & $\begin{array}{c}\text { Pre } \\
\text { Tx---post } \\
\text { EBRT diff. }\end{array}$ & $\begin{array}{c}\text { Pre Tx } \\
---P o s t ~ T x \\
\text { diff. }\end{array}$ \\
\hline $\begin{array}{c}\text { Tumor } \\
\text { diameter(mm) }\end{array}$ & $46.50 \pm 10.15$ & $24.97 \pm 7.33$ & $17.23 \pm 7.42$ & $44.90 \pm 15.26$ & $62.73 \pm 14.96$ & $<0.001 * *$ & $<0.001^{* *}$ \\
\hline Volume $\left(\mathrm{cm}^{3}\right)$ & $33.03 \pm 12.37$ & $5.94 \pm 2.19$ & $2.35 \pm 1.81$ & $77.23 \pm 15.74$ & $91.90 \pm 7.78$ & $<0.001 * *$ & $<0.001^{* *}$ \\
\hline $\begin{array}{c}\text { Tumor } \mathrm{ADC} \\
\left(\mathrm{x} 10^{-3} \mathrm{~mm}^{2} / \mathrm{s}\right)\end{array}$ & $1.05 \pm 0.18$ & $1.33 \pm 0.15$ & $1.52 \pm 0.17$ & $28.83 \pm 17.79$ & $48.10 \pm 20.54$ & $<0.001 * *$ & $<0.001 * *$ \\
\hline
\end{tabular}

\subsection{Statistical Analysis}

Descriptive and inferential statistical analysis has been carried out in the present study. Results on continuous measurements are presented on Mean \pm SD (Min-Max) and results on categorical measurements are presented in Number (\%). Significance is assessed at $5 \%$ level of significance. The following assumptions on data are made,

1. Dependent variables should be normally distributed,

2. Samples drawn from the population should be random, and

3. Cases of the samples should be independent.

Analysis of variance (ANOVA) has been used to find the significance of study parameters between three or more groups of patients, Student $t$ test ( two tailed, independent) has been used to find the significance of study parameters on continuous scale between two groups (Inter group analysis) on metric parameters. Pearson correlation has been used between $\mathrm{ADC}$ and $\% \mathrm{ADC}$ changes with \% tumor size and volume response.

Statistical software: The Statistical software namely SAS 9.2, SPSS 15.0, Stata 10.1, MedCalc 9.0.1, Systat 12.0 and R environment ver.2.11.1 were used.

\section{Results}

\subsection{Response Evaluation.}

The pretreatment mean diameter of tumors was $46.50 \pm 10.15$, post EBRT $24.97 \pm 7.33$ and post treatment $17.23 \pm 7.42 \mathrm{~mm}$. A significant difference was found between pretreatment and post EBRT or post treatment tumor diameter (both $\mathrm{P}<0.001$ ).

The mean tumor volumes were $33.03 \pm 12.37$ at pretreatment, $5.94 \pm 2.19$ at post EBRT and $2.35 \pm 1.81 \mathrm{~cm}^{3 \text { at }}$ post treatment, respectively. A statistically significant difference was found between pretreatment and post EBRT and between pretreatment and post treatment tumor volumes (both $\mathrm{P}<0.001$ ).

The mean tumor ADCs were $1.05 \pm 0.18$ at pretreatment, $1.33 \pm 0.15$ at post EBRT and $1.52 \pm 0.17 \times 10^{-3} \mathrm{~mm}^{2} / \mathrm{s}$ at post treatment respectively. A significant difference was found between pretreatment and post EBRT and between pretreatment and post treatment ADCs (both $\mathrm{P}<0.001$ )

The post EBRT and final size responses were $44.90 \%$ and $62.73 \%$ respectively.

The post EBRT and final volume responses were $77.23 \%$ and $91.90 \%$ respectively.

The mean change in tumor ADCs at post EBRT and post treatment were $28.83 \%$ and $48.10 \%$ respectively. (Table 1 )

\subsection{Correlation of Tumor ADC and \% Tumor ADC Change with Final Response}

We found statistically significant and moderate positive linear correlation between tumor ADCs at different treatment stages and the final size reduction. There was a statistically suggestive significance between the tumor ADC at pretreatment and final volume reduction. There was a statistically significant and moderate positive linear correlation between the tumor ADCs at post EBRT and post treatment and the final volume reduction.

The changes in the tumor ADCs at post EBRT had a significant and moderate positive correlation with the final tumor size and volume reductions. (Table 2, Table 3)

\subsection{Treatment Response}

Two of 30 patients $(6.7 \%)$ showed no residual tumor on both $\mathrm{T} 2 \mathrm{~W}$ and DW images after the completion of therapy indicating complete response (Fig 1). 17 patients(56.7\%) showed good response with $>60 \%$ final size reduction (Fig 2) and 11 patients $(36.7 \%)$ showed partial response (Fig 3 ) with $<60 \%$ final size reduction after completion of therapy.

Table 2. Correlation of Tumour ADC with final \% size and volume reduction

\begin{tabular}{|c|c|c|c|c|c|c|}
\hline \multirow{3}{*}{ Pair } & \multicolumn{6}{|c|}{ Pearson correlation } \\
\hline & \multicolumn{2}{|c|}{ Pre-Tx } & \multicolumn{2}{|c|}{ Post EBRT } & \multicolumn{2}{|c|}{ Post-Tx } \\
\hline & r value & p value & r value & p value & r value & p value \\
\hline Tumor ADC vs \% final size reduction & 0.391 & $0.033 *$ & 0.402 & $0.028 *$ & 0.389 & $0.034 *$ \\
\hline Tumor ADC vs \% final volume reduction & 0.335 & $0.070+$ & 0.393 & $0.032 *$ & 0.467 & $0.009 * *$ \\
\hline
\end{tabular}


Table 3. Correlation of Tumor ADC $\%$ change with $\%$ tumor size reduction

\begin{tabular}{|c|c|c|}
\hline \multirow{2}{*}{ Pair } & \multicolumn{2}{|c|}{ Pearson correlation } \\
\hline & r value & p value \\
\hline$\%$ post EBRT ADC change vs $\%$ post EBRT -size response & 0.035 & 0.80 \\
\hline$\%$ post EBRT ADC change vs $\%$ final size response & 0.489 & $0.04 *$ \\
\hline$\%$ final ADC change vs $\%$ final size response & 0.027 & 0.764 \\
\hline
\end{tabular}
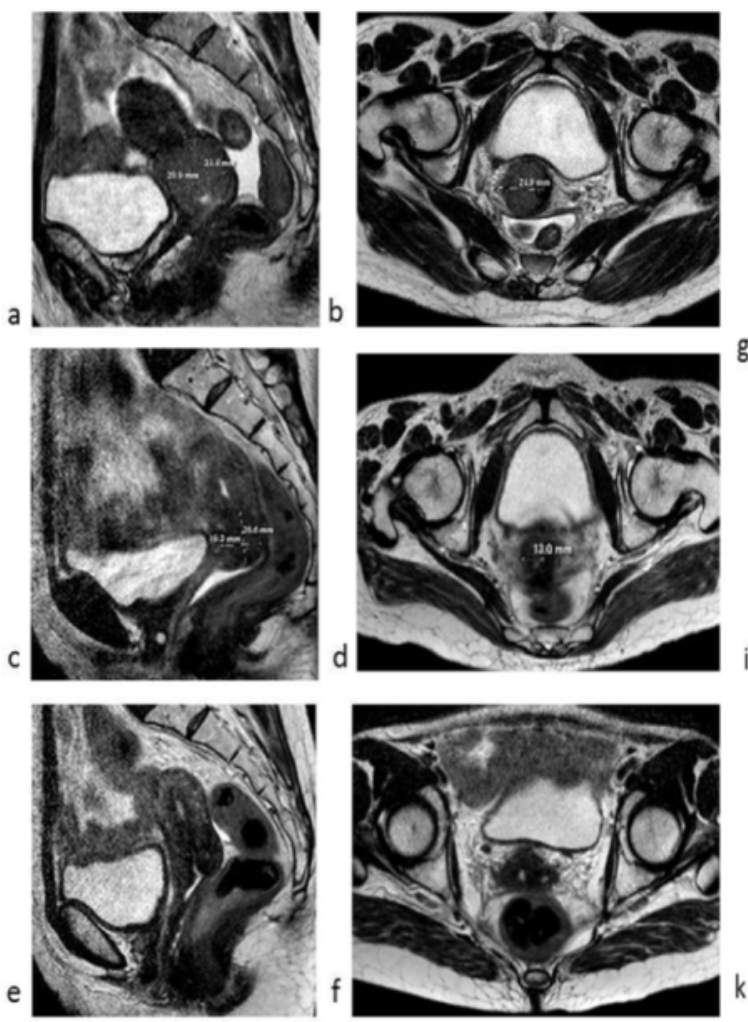
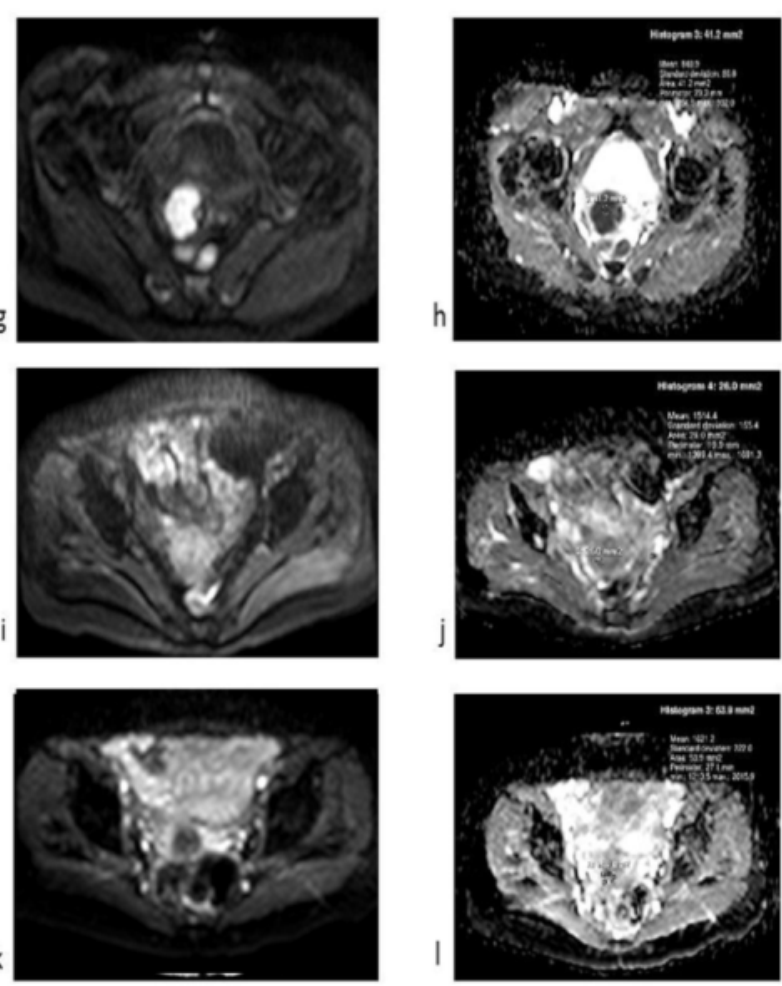

Figure 1. Conventional MR images (a-f) and DW images (g-l) of a woman with uterine cervical carcinoma showing complete response to chemo radiation therapy. The sagittal and axial T2W images at pre-Tx (a, b), Post EBRT (c, d) and post-Tx (e, f) illustrates consecutive reduction in the tumor size. On corresponding ADC maps, the tumor illustrates serial increase in the ADC values with 0.8 at pre-Tx (g, h), 1.5 at Post EBRT (i, j) and 1.6 x 10-3 mm2/s at post-Tx(k, l). 

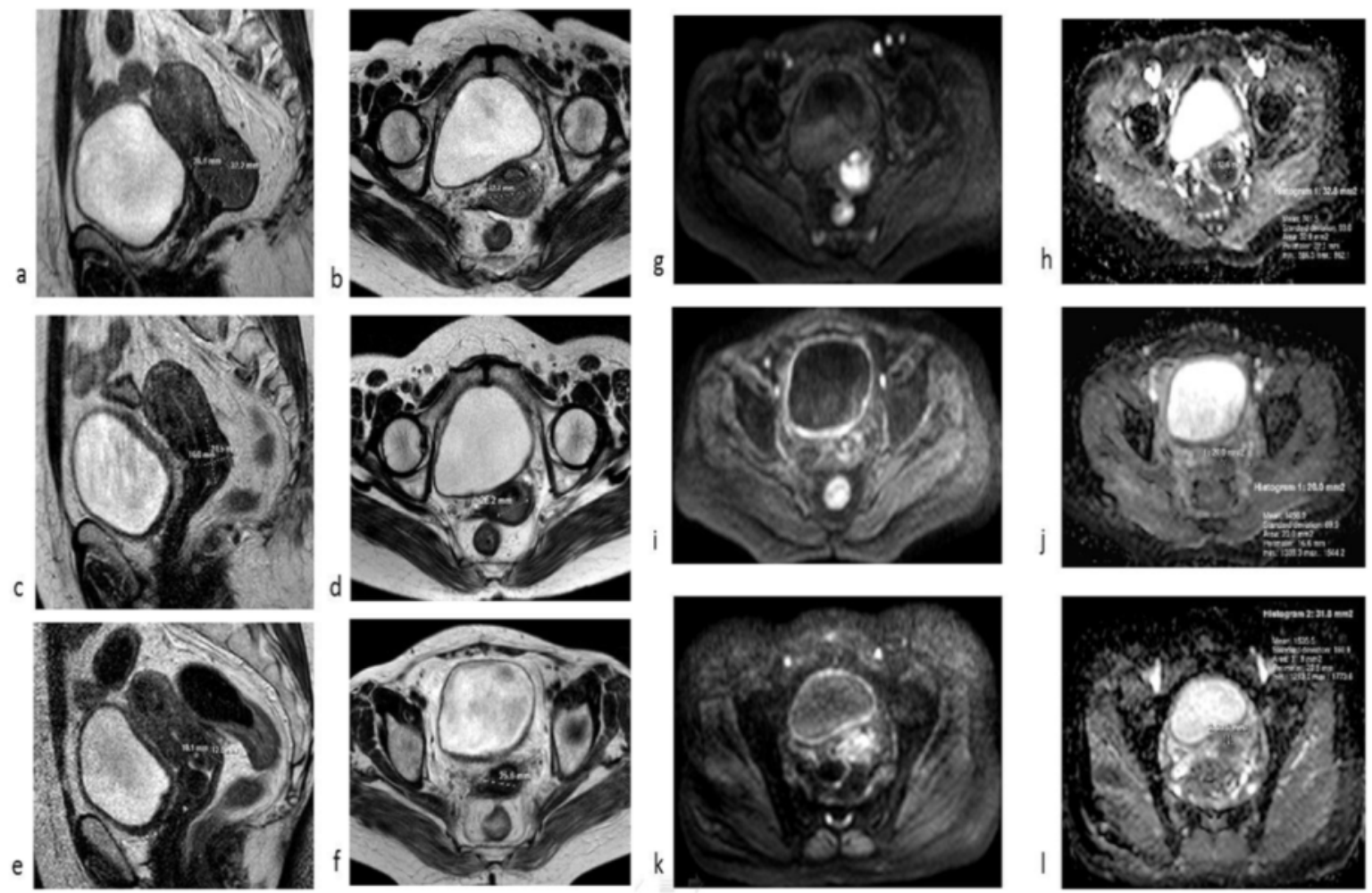

Figure 2. Conventional MR images (a-f) and DW images (g-l) of a woman with uterine cervical carcinoma showing good response to chemo radiation therapy. The sagittal and axial T2W images at pre-Tx (a, b), Post EBRT (c, d) and post-Tx (e, f) illustrates consecutive reduction in the tumor size. On corresponding ADC maps, the tumor illustrates serial increase in the ADC values with 0.7 at pre-Tx (g, h), 1.4 at Post EBRT (i, j) and 1.5 x 10-3 mm2/s at post-Tx(k, l).
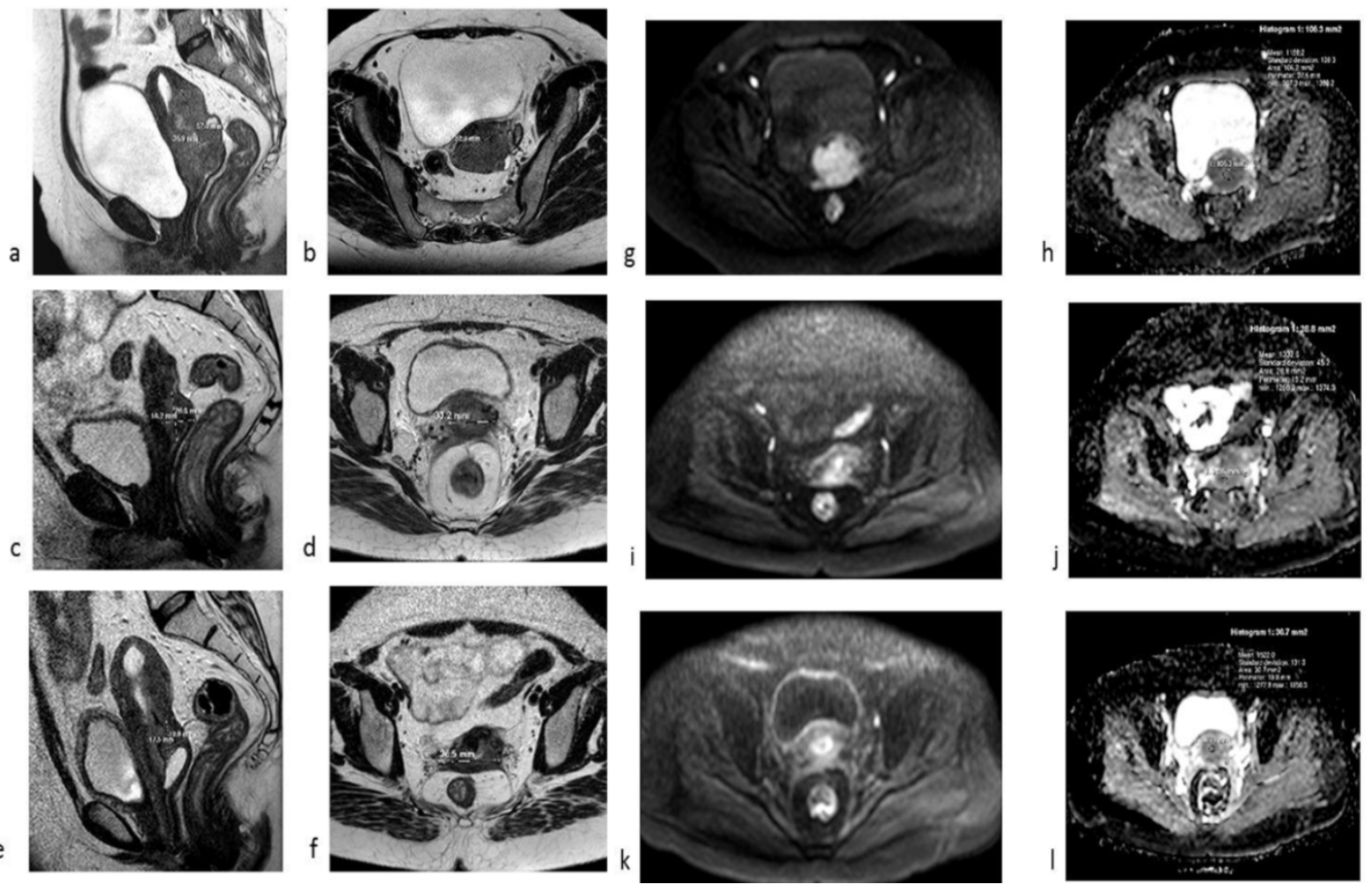

Figure 3. Conventional MR images (a-f) and DW images (g-l) of a woman with uterine cervical carcinoma showing partial response to chemo radiation therapy. The sagittal and axial T2W images at pretreatment (a, b), Post EBRT (c, d) and post-treatment (e, f) illustrates consecutive reduction in the tumor size. On corresponding ADC maps, the tumor illustrates serial increase in the ADC values with 1.1 at pre-Tx (g, h), 1.3 at Post EBRT (i, j) and 1.5 x 10-3 $\mathrm{mm} 2 / \mathrm{s}$ at post-Tx $(\mathrm{k}, \mathrm{l})$. Post EBRT 
Table 4. Comparison of tumor ADC and $\%$ ADC change between the response groups

\begin{tabular}{|c|c|c|c|c|c|}
\hline \multirow{2}{*}{$\begin{array}{c}\text { Tumor ADC } \\
\left(\mathbf{x 1 0} \mathbf{m m}^{2} / \mathbf{s}\right)\end{array}$} & \multicolumn{3}{|c|}{ Response } & \multirow{2}{*}{ Total } & \multirow{2}{*}{ P value } \\
\cline { 2 - 5 } & $\begin{array}{c}\text { Complete } \\
\text { Response }\end{array}$ & Good Response & $\begin{array}{c}\text { Partial } \\
\text { Response }\end{array}$ & $1.05 \pm 0.18$ & $0.030^{*}$ \\
\hline Pre-treatment & $1.35 \pm 0.02$ & $1.04 \pm 0.17$ & $1.01 \pm 0.15$ & $1.33 \pm 0.15$ & $0.042^{*}$ \\
\hline Post EBRT & $1.55 \pm 0.05$ & $1.34 \pm 0.11$ & $1.27 \pm 0.18$ & $1.52 \pm 0.17$ & $0.080+$ \\
\hline Post treatment & $1.66 \pm 0.01$ & $1.56 \pm 0.10$ & $1.43 \pm 0.23$ & $28.83 \pm 7.80$ & 0.444 \\
\hline \% ADC change post EBRT & $15.00 \pm 0.00$ & $31.53 \pm 9.78$ & $27.18 \pm 5.46$ & $48.10 \pm 10.54$ & 0.267 \\
\hline
\end{tabular}

\subsection{Comparison of Tumor ADC and \% ADC Change between the Response Groups}

The pre, post EBRT and post treatment mean tumor ADC of the complete response (CR) and good response (GR) groups were significantly larger than that of partial response (PR) group.

Post EBRT and final treatment ADC \% change of the partial response group was greater than that of complete response (CR) group, however showed no statistical significance. (Table 4)

\subsection{Comparative Analysis of Tumor ADC with Respect to Parametrial, Vaginal, Lymph Node Involvement and FIGO Stage}

The tumor ADC values of patients with parametrial involvement and vaginal invasion were lower than those without extensions on pretreatment evaluation, but revealed no statistically significant association.

The tumor ADC values of patients with lymph nodal involvement is higher than those without nodal involvement at pretreatment evaluation however without statistically significant association .The tumor ADC values of patients with early FIGO stages ( stage II) is higher those with late FIGO stages (stage III / IV) on pretreatment evaluation but there was no statistically significant association.

\section{Discussion}

Concurrent chemo radiation therapy (CCRT) has been widely accepted for the primary treatment of uterine cervical carcinoma. However, once treatment fails further treatment options become limited and result in poor outcome. If the prediction of treatment response can be achieved before or during treatment, it could be effectively used in changing therapeutic strategies to avoid toxicity or negative side effects of ineffective therapy. Furthermore, if pre-treatment imaging was able to identify patients at high risk for disease recurrence before CCRT, better treatment could be accomplished by conducting more intensive follow-ups or considering a choice of clinical trials.

Diffusion-weighted magnetic resonance imaging (DWI) has been recently introduced in cancer diagnostics and has widened the diagnostic possibilities of MRI. DWI is a functional imaging technique that analyses differences in the extracellular movement of water protons to discriminate between tissues of varying cellularity [5]. DWI has shown its potentially beneficial role for the detection and characterization of malignant tumours. Thus, this technique allows for quantification of diffusion by calculating the apparent diffusion coefficient (ADC). The ADC, a quantitative parameter measured on DWI, may provide useful information regarding tumour cellularity, tumour aggressiveness, subtype characterization and cancer treatment response $[4,6]$.

In our study, we measured the serial apparent diffusion coefficient (ADC) values for cervical cancer before, during and after chemoradiotherapy and correlated the ADC values with loco regional outcome. The purpose of this study was to investigate the role of diffusion-weighted imaging (DWI) in predicting and monitoring chemo radiotherapy response in patients with uterine cervical carcinoma.

The uterine cervical tumor demonstrates high signal intensity on DW images and low signal intensity on ADC maps. Several studies have found that the mean ADC value of cervical tumor is significantly lower than that of normal cervical tissue. Naganawa et al. [7]applied DWI to cervical carcinoma and found that the mean ADC value of cervical cancer lesions $\left(1.09 \pm 0.20 \times 10^{-3} \mathrm{~mm}^{2} / \mathrm{s}\right)$ was lower than that of normal cervix $\left(1.79 \pm 0.24 \times 10^{-3} \mathrm{~mm}^{2} / \mathrm{s}\right)$, and increased after therapy $\left(1.48 \pm 0.23 \times 10^{-3} \mathrm{~mm}^{2} / \mathrm{s}\right)^{(9)}$. McVeigh et al. [3] reported that the median ADC of cervical cancers $\left(1.09 \pm 0.20 \times 10^{-3} \mathrm{~mm}^{2} / \mathrm{s}\right)$ was lower than that of control cervical tissue $\left(2.09 \pm 0.46 \times 10^{-3} \mathrm{~mm}^{2} / \mathrm{s}\right)$ with very little overlap $^{(8)}$. In a study by Chen et al[4], the ADC values of normal cervical tissue, cervical carcinoma before and after chemoradiotherapy, were measured respectively and found that the mean ADC value of cervical carcinoma $\left(1.013 \pm 0.094 \times 10^{-3} \mathrm{~mm}^{2} / \mathrm{s}\right)$ in 22 patients was lower than that of normal cervical tissue $\left(1.593 \pm 0.151 \times 10^{-3} \mathrm{~mm}^{2} / \mathrm{s}\right)$ and increased following the completion of therapy $\left(1.436 \pm 0.129 \times 10^{-3} \mathrm{~mm}^{2} / \mathrm{s}\right)^{(1)}$.

More recently Hyun et al [8],reported that the mean tumor ADCs were $0.88 \pm 0.12 \times 10^{-3} \mathrm{~mm}^{2} / \mathrm{s}$ at pre -treatment, $1.30 \pm 0.17 \times 10^{-3} \mathrm{~mm}^{2} / \mathrm{s}$ at post EBRT and $1.47 \pm 0.17 \times 10^{-3} \mathrm{~mm}^{2} / \mathrm{s}$ at post treatment, respectively which showed a significant difference $(\mathrm{P}<0.001)$. A significant difference between pretreatment and mid -treatment, or between pre-treatment and post treatment or between post 
EBRT and post -treatment was found (all, <0.001).

The results of our study are similar to the above discussed studies with the mean tumor ADCs of $1.05 \pm 0.18$ at pre-treatment, $1.33 \pm 0.15$ at post EBRT and $1.52 \pm 0.17$ $\times 10^{-3} \mathrm{~mm}^{2} / \mathrm{s}$ at post-treatment respectively. A significant difference was found between pre-treatment and post EBRT and between pre-treatment and post treatment (both $\mathrm{P}<0.001$ ). The reason for reduction in $\mathrm{ADC}$ value within the tumor is due to hypercellularity within the malignant tissue causing restriction of diffusion of water molecules .The increase in $\mathrm{ADC}$ values following chemoradiation therapy may be presumably due to cellular apoptosis and increase in extracellular space resulting in increased water diffusion. However the cause for the ADC values to be still lower than that of normal cervical tissue was the presence of edema, hyaline degeneration and granulation tissue in the cervical tissue after therapy.

Hyun et al [8], reported the mean change in tumor ADCs of $51.7 \%(20-80 \%)$ at mid -treatment. This is similar to our value of $28.83 \%$ at mid-treatment. Our study also calculated the mean change in tumor ADCs at post-treatment of $48.10 \%$.

Harry et al. [9] in a recent study has evaluated DW-MRI as an early response indicator in women receiving chemoradiation for advanced cervical cancer It was found that the ADC values after 2 weeks of therapy correlated with eventual MR response $(p=0.048)$ and clinical response $(p=$ $0.009)$, as did the change in ADC values after 2 weeks of therapy ( $p=0.01$ for MR response; $p=0.03$ for clinical response). Lui et al. [10], found a negative correlation between pretreatment ADCs and percentage size reduction after 2 months of chemoradiation ( $\mathrm{p}=0.016$ ). The percentage ADC change after 1 month correlated positively with percentage size reduction after 2 months of therapy $(p=0.021)$. However, Hyun et al. [8] found that tumor ADCs at post EBRT had a significant correlation with the final size response but pre-treatment $\mathrm{ADC}$ values were not associated with final size response. Both pre and post EBRT ADCs did not show significant correlation with the final tumor volume response. The changes in tumor ADCs at mid-treatment had a significant correlation with the final size response; however mid-treatment ADC changes were not associated with the final volume response.

Correlations obtained in our study were consistent with that of Harry and Hyun with a significant and moderate positive linear correlation between the tumor ADCs at pre-treatment, post EBRT and post-treatment with the final size reduction (Pearson coefficient $=0.391,0.402,0.389$ respectively and $\mathrm{P}$ value $=0.033,0.028,0.034$ respectively).

The tumor ADC at pre-treatment showed statistically suggestive significance with final volume response (Pearson coefficient $=0.335$ and $\mathrm{P}$ value $=0.070$ ). The tumor ADCs at post EBRT and post-treatment had significant and moderate positive linear correlation with the final volume response (Pearson coefficient $=0.393$ and 0.467 respectively and $\mathrm{P}$ value $=0.032$ and 0.009 respectively).

A significant and moderate positive correlation was found between post EBRT tumor ADC changes and the final tumor size and volume responses (Pearson coefficient $=0.489$ and 0.375 respectively, both $\mathrm{p}=0.04$ ).

In our study, two of 30 patients $(6.7 \%)$ showed no residual tumor on both $\mathrm{T} 2 \mathrm{~W}$ and $\mathrm{DW}$ images after the completion of therapy indicating complete response. 17 patients $(56.7 \%)$ showed good response with $>60 \%$ final size response and 11 patients (36.7\%) showed partial response with $<60 \%$ final size response after completion of therapy.

Liu et al [10], reported the pre-treatment tumor diameter of the CR group was larger than the PR group, but there was no significant difference between them (t $1 / 4$ 1.935, p $1 / 4$ 0.074). Another study by Suk Hee Heo et al [11], found borderline significance of mean tumor diameter with tumor recurrence $(p=0.055)$. The mean tumor diameter was $5.7(3.9-7.5) \mathrm{cm}$ in recurrence group and $4.5(2-9.3) \mathrm{cm}$ in no recurrence group.

In our study, the pretreatment tumor diameter and volume of the complete response (CR) and good response (GR) groups were larger than that of partial response (PR) group, but there was no significant difference between them similar to the study by Liu.

We also found that the mid and post treatment tumor diameters and volumes of the complete response (CR) and good response (GR) groups were significantly lower than that of partial response (PR) group. Post EBRT and post treatment \% size/ volume reduction of the complete response (CR) and good response(GR) groups were significantly greater than that of partial response (PR) group ( $p=0.010$ and $\mathrm{p}<0.001$ respectively).

In study by Liu et al [10], the pretreatment mean ADC of patients with CR (Complete response) was significantly lower than those with PR (Partial response) group. The pretreatment ADC value of the SD (Stable disease) case was higher than that of the CR group and slightly lower than that of the PR group. For the CR group, the mean ADC value after 1 month of therapy increased to a great extent and it was statistically significantly higher than the pretreatment ADC value $(p<0.001)$. The mean percentage ADC changes of tumor after 1 month of treatment were $56.8 \%$ for CR, 28.8\% for PR and $24.2 \%$ for SD. Hyun et al [8] demonstrated no statistical difference in mean tumor ADCs at pretreatment between responder and non-responder groups. Suk Hee Heo et al [11],found the values of mean ADC (mADC) and $75^{\text {th }}$ percentile ADC (ADC75) were significantly higher in the recurrence group as compared to the no recurrence group ( $p$ $=0.043$ and $p=0.008$, respectively).

However in our study, the pre, mid and post treatment mean tumor ADCs of the complete response (CR) and good response (GR) groups were significantly larger than that of partial response (PR) group $(\mathrm{p}=0.030, \mathrm{p}=0.042$ and $\mathrm{p}=0.004)$. The mid and final treatment $\%$ ADC changes of the partial response group was greater than that of complete response (CR) group and lesser than that of good response $(\mathrm{GR})$ groups $(\mathrm{CR}<\mathrm{PR}<\mathrm{GR})$, however showed no statistical significance $(\mathrm{p}=0.444$ and $\mathrm{p}=0.267)$.

Nakamura et al [12] indicated that mean ADC of the 
tumor was lower in FIGO stages IIb-IVa and with parametrial, vaginal, pelvic lymph node involvement. The pretreatment ADC mean of the primary tumor showed significant associations with the FIGO stage and parametrial involvement $(p<0.001$ and $p=0.002$ respectively). The post treatment mean $\mathrm{ADC}$ of the primary tumor was significantly associated with parametrial involvement $(\mathrm{p}=0.045)$. The pre and post treatment mean ADC were not statistically associated with vaginal invasion and pelvic lymph node metastases. Mc Veigh et al. [3] showed that median ADC of cervical cancer was significantly lower in FIGO stages T1B/T2a compared to T2b and T3/T4. Payne et al showed that there was no significant difference in tumor ADCs when separated by other characteristics like tumor type and lymph node metastases.

Results of our study showed that the tumor ADC values of patients with parametrial and vaginal involvement was lower than those without involvement at all treatment stages, but there was no statistically significant association. The tumor $\mathrm{ADC}$ values of patients with lymph nodal involvement was higher than those without nodal involvement but there was no statistically significant association. The tumor ADC values of patients with early FIGO stages ie II was higher those with late FIGO stages ie III / IV at pretreatment evaluation but there was no statistically significant difference.

Limitations: This study had some limitations. It was a single center based study with a small study group. Also the follow up was limited to 6 months with the short duration of study of 2 yrs.

\section{Conclusions}

The addition of diffusion weighted imaging to the conventional magnetic resonance imaging protocol allows precise assessment of uterine cervical carcinoma, from lesion detection and characterization to staging of tumor. When used in conjunction with apparent diffusion coefficient mapping, diffusion weighted imaging is a powerful tool with the potential for early and accurate prediction, assessment and monitoring of response to chemo radiation therapy.

Our study concluded that:

- The uterine cervical tumor demonstrates restriction on DW images and significantly lower values on ADC maps.

- There was a serial increase in the tumor ADC values along with corresponding decrease in the tumor diameters and volumes during the chemo radiation therapy.

- Tumors with high diffusion values will respond better to therapy than low diffusion values. Post EBRT patients with greater tumor ADC changes, greater size and volume reduction show better response to therapy. Hence the pretreatment tumor ADC values, mid treatment tumor ADC changes, tumor size and volume responses are significant prognostic predictors of treatment response.

- However ADC mapping is less useful in predicting the extension and staging of uterine cervical carcinoma.

\section{Advances in Knowledge}

- There was a serial increase in the tumor ADC values along with corresponding decrease in the tumor diameters and volumes during the chemo radiation therapy.

- Tumors with high diffusion values will respond better to therapy than low diffusion values. Post EBRT patients with greater tumor $\mathrm{ADC}$ changes, greater size and volume reduction show better response to therapy. Hence the pretreatment tumor ADC values, mid treatment tumor ADC changes, tumor size and volume responses are significant prognostic predictors of treatment response.

- However ADC mapping is less useful in predicting the extension and staging of uterine cervical carcinoma.

\section{Implications for Patient Care}

- The addition of diffusion weighted imaging to the conventional magnetic resonance imaging protocol allows precise assessment of uterine cervical carcinoma, from lesion detection and characterization to staging of tumor.

- When used in conjunction with apparent diffusion coefficient mapping, diffusion weighted imaging is a powerful tool with the potential for early and accurate prediction, assessment and monitoring of response to chemo radiation therapy.

\section{REFERENCES}

[1] Evis Sala, Andrea Rockall, Deepa Rangarajan, Rahel A. The role of dynamic contrast enhanced and diffusion weighted magnetic resonance imaging in the female pelvis. European Journal of Radiology 76 (2010) $367-385$.

[2] Wang J, Mayr N, Zang D, [et al.]. Sequential magnetic resonance imaging of cervical cancer: the predictive value of absolute tumor volume and regression ratio measured before, during and after radiation therapy. Cancer. 2010, 116, 5093-5101.ynecol Oncol 2010; 116: 246-252.

[3] McVeigh PZ, Syed AM, Milosevic M, Fyles A, Haider MA. Diffusion-weighted MRI in cervical cancer. Eur Radiol 2008; 18: 1058-64.

[4] Chen Jianyu, ZhangYun, LiangBiling, YangZehong. The utility of diffusion-weighted MR imaging in cervical cancer. European Journal of Radiology 74 (2010) 101-106.

[5] Bammer, R. 2003. Basic principles of diffusion-weighted imaging. Eur. J. Radiol. 45:169-184. 
[6] Brian D. Ross, Craig J. Galbán, and Alnawaz Rehemtulla. DW-MRI Assessment of Cancer response to Chemoradiation. Diffusion-weighted MR imaging, applications in the body, 10 (2010): 157-171.

[7] Naganawa S, Sato C, Kumada H, Ishigaki T, Miura S, Takizawa O. Apparent dif-fusion coefficient in cervical cancer of the uterus: comparison with the normal uterine cervix. Eur. Radiol 2005; 15: 71-8.

[8] Hyun Su Kim, Chan Kyo Kim, Byung Kwan. Evaluation of therapeutic response to concurrent chemoradiotherapy in patients with cervical cancer using diffusion weighted MR imaging. Journal of Magnetic Resonance Imaging 37(2013):187-193.

[9] Harry VN, Semple SI, Gilbert FJ, and DE Parkin.(2008) Diffusion-weighted magnetic resonance imaging in the early detection of response to chemoradiation in cervical cancer.
GynecolOncol 111: 213-220.

[10] Liu Y, Bai R, Sun H, et al. Diffusion-weighted imaging in predicting and monitoring the response of uterine cervical cancer to combined chemoradiation. Clinical journal of Radiology 2009; 64(11):1067-74.

[11] Suk Hee Heo, MD1, Sang Soo Shin, MD2, 3, Jin Woong Kim et al. Pre-Treatment Diffusion-Weighted MR Imaging for Predicting Tumor Recurrence in Uterine Cervical Cancer Treated with Concurrent Chemoradiation: Value of Histogram Analysis of Apparent Diffusion Coefficients. Korean J Radiol 2013; 14(4):616-625.

[12] Nakamura, K., I. Joja, T. Nagasaka, C. Fukushima, T. Kusumoto, N. Seki, et al. 2012. The mean apparent diffusion coefficient value (ADC (mean)) on primary cervical cancer is a predictive. 